nephron

Practice
Nephron

DOI: $10.1159 / 000489897$
Received: March 13, 2018

Accepted after revision: May 7, 2018

Published online: May 23, 2018

\title{
Disparities in Chronic Kidney Disease Prevalence among Males and Females in 195 Countries: Analysis of the Global Burden of Disease 2016 Study
}

\author{
Boris Bikbov ${ }^{a}$ Norberto Perico ${ }^{a}$ Giuseppe Remuzzi ${ }^{a-c}$ \\ on behalf of the GBD Genitourinary Diseases Expert Group

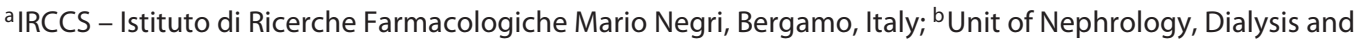 \\ Transplantation, Azienda Socio-Sanitaria Territoriale Papa Giovanni XXIII, Bergamo, Italy; ${ }^{C}$ L. Sacco Department of \\ Biomedical and Clinical Sciences, University of Milan, Milan, Italy
}

\section{Keywords \\ Chronic kidney disease - Dialysis - Gender disparities - Global burden of disease - Morbidity . \\ Prevalence $\cdot$ Transplantation}

\begin{abstract}
Background: Chronic kidney disease (CKD) imposes a substantial burden on health care systems. There are some especially vulnerable groups with a high CKD burden, one of which is women. We performed an analysis of gender disparities in the prevalence of all CKD stages and renal replacement therapy (defined as impaired kidney function [IKF]) in 195 countries. Methods: We used estimates produced by the Global Burden of Disease (GBD) Study 2016 revision using a Bayesian-regression analytic tool, DisMoD-MR 2.1. Data on gross domestic product based on purchasing power parity per capita (GDP PPP) was obtained via the World Bank International Comparison Program database. To estimate gender disparities, we calculated the male:female all-age prevalence rate ratio for each IKF condition. Results: In 2016, the global number of individuals with IKF reached 752.7 million, including 417.0 million females and 335.7 million males. The most prevalent form of IKF in both groups was albuminuria
\end{abstract}

with preserved glomerular filtration rate. Geospatial analysis shows a very heterogeneous distribution of the male:female ratio for all IKF conditions, with the most prominent contrast found in kidney transplant patients. The median male:female ratio varies substantially according to GDP PPP quintiles; however, countries with different economic states could have similar male:female ratios. A strong correlation of GDP PPP with dialysis-to-transplant ratio was found. Conclusions: The GBD study highlights the prominent gender disparities in CKD prevalence among 195 countries. The nature of these disparities, however, is complex and must be interpreted cautiously taking into account all possible circumstances.

(c) 2018 S. Karger AG, Basel

\section{Introduction}

Chronic kidney disease (CKD) is one of the most rapidly growing non-communicable diseases (NCD) and imposes a substantial mortality and morbidity burden [1-3]. The Global Burden of Disease, Injuries and Risk Factors Study (GBD) is one of the most important projects undertaken in modern epidemiology, and the GBD

\section{KARGER}

(c) 2018 S. Karger AG, Basel

E-Mail karger@karger.com

www.karger.com/nef
Dr. Boris Bikbov

IRCCS - Istituto di Ricerche Farmacologiche Mario Negri

Via G.B. Camozzi 3

Ranica, IT-24020 Bergamo (Italy)

E-Mail boris.bikbov@marionegri.it 
2016 revision allowed to produce estimates for the global prevalence of all CKD stages $[1,3]$. Among the summary estimates for CKD, there are some particularly vulnerable groups with high disease prevalence and one of these is women. Notably, CKD in women has profound consequences for global health, since it substantially increases the risk of premature delivery, infants who are small for their gestational age and low birth weight [4], which predispose the next generation to a higher risk for NCD $[5,6]$. In 2018, the celebration of World Kidney Day is dedicated to women's health [7], with the major aims being to increase awareness of CKD in the female population and improve kidney health care for women. In this article, we explore global gender disparities in CKD prevalence based on data from the GBD 2016 Study.

\section{Materials and Methods}

To retrieve data on CKD prevalence, the GBD Genitourinary Expert Group (GUiDEG) and the Institute of Health Metrics and Evaluations performed several rounds of systematic review, covering PubMed and EMBASE, as well as including literature obtained by manual search. Additional data from unpublished data sources, including hospital discharge records, end-stage kidney disease registries, and so on were provided by the geographically distributed GBD 2016 CKD collaborators. Data sources used for CKD burden modelling can be explored in an online data source tool http://ghdx.healthdata.org/gbd-2016/data-input-sources. Estimates of CKD prevalence were produced using a Bayesian-regression analytic tool (DisMoD-MR 2.1). More details on GBD methods are described elsewhere $[1,2]$. Data on gross domestic product based on purchasing power parity per capita (GDP PPP) was obtained via the World Bank International Comparison Program database [8].

The GBD Study considers 6 categories of patients with CKD based on albuminuria and glomerular filtration rate (GFR) grades according to the KDIGO classification [9], or receipt of renal replacement therapy (RRT):

- Albuminuria with normal GFR (estimated GFR [eGFR] $\geq 60 \mathrm{~mL} / \mathrm{min} / 1.73 \mathrm{~m}^{2}$ ) - refers to KDIGO A2-A3 G1-G2 grades.

- CKD stage 3 (eGFR $30-59 \mathrm{~mL} / \mathrm{min} / 1.73 \mathrm{~m}^{2}$ ) - refers to KDIGO G3 GFR grade independent of albuminuria.

- CKD stage 4 (eGFR $\left.15-29 \mathrm{~mL} / \mathrm{min} / 1.73 \mathrm{~m}^{2}\right)$ - refers to KDIGO G4 GFR grade independent of albuminuria.

- CKD stage $5\left(\mathrm{eGFR}<15 \mathrm{~mL} / \mathrm{min} / 1.73 \mathrm{~m}^{2}\right)$ not on RRT - refers to KDIGO G5 GFR grade independent of albuminuria.

- Maintenance dialysis.

- Kidney transplantation.

Considering the aforementioned differences between the CKD categories defined in KDIGO and GBD, and the need to map kidney disease as a risk factor for cardiovascular disease, the GBD terminology introduced the term "impaired kidney function" (IKF), with 6 IKF conditions determined by the above-mentioned categories of CKD patients.

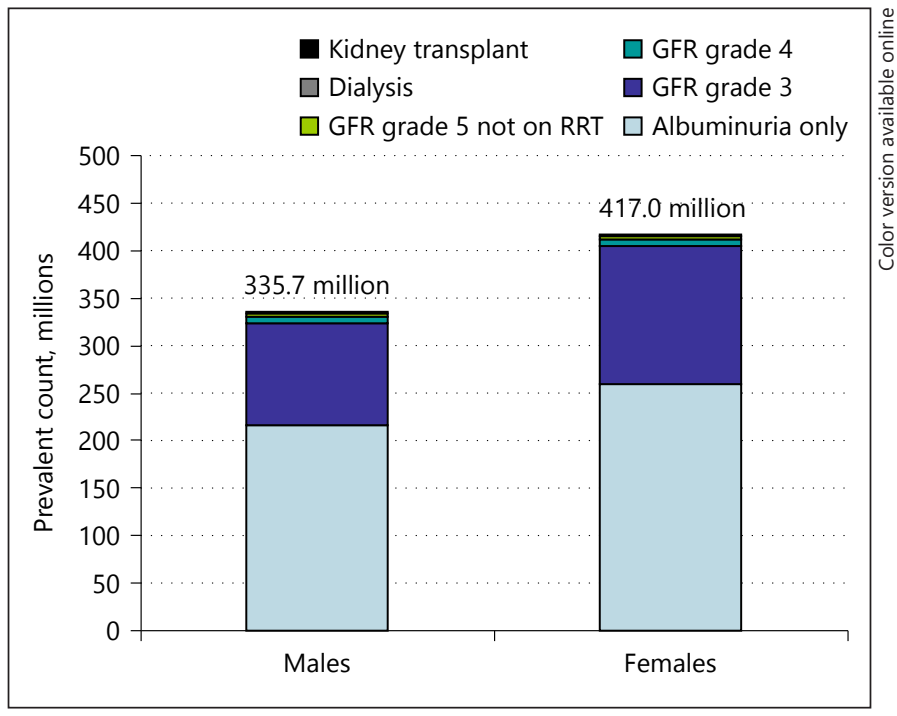

Fig. 1. Global number of persons with CKD in 2016. CKD, chronic kidney disease; GFR, glomerular filtration rate; RRT, renal replacement therapy.

To estimate gender disparities, we calculated the male:female all-age prevalence rate ratio for each IKF condition for 195 countries in all world regions for the year 2016. Taking into account the sensitivity of this ratio in case of small numbers in either numerator or denominator, for the RRT modalities, we calculated the male:female ratio both with all country data and separately with countries having substantial numbers of RRT patients.

Differences in continuous variables between groups were compared by Wilcoxon's rank-sum test. Correlations between studied parameters were estimated by the Spearman rho. A $p$ value less than 0.05 was considered statistically significant. The analyses were performed with $\mathrm{R}$ (version 3.4.3).

\section{Results}

\section{Global Estimates}

In 2016, the GBD estimates that a global number of individuals with IKF accounted for 752.7 million, with 417.0 million females and 335.7 million males (Fig. 1). The most prevalent form of IKF in both gender groups was albuminuria with preserved GFR, which globally was estimated to affect 260.1 million females (62.4\% from IKF in females) and 216.7 million males (64.5\% from IKF in males). Another 145.2 million females (34.8\% from IKF in females) and 107.6 million males (32.1\% from IKF in males) had GFR grade 3. More advanced CKD stages were less prevalent: GFR grade 4 affected 6.8 million females and 6.2 million males (1.6 and $1.8 \%$ from IKF respectively); GFR grade 5 without RRT was estimated to 


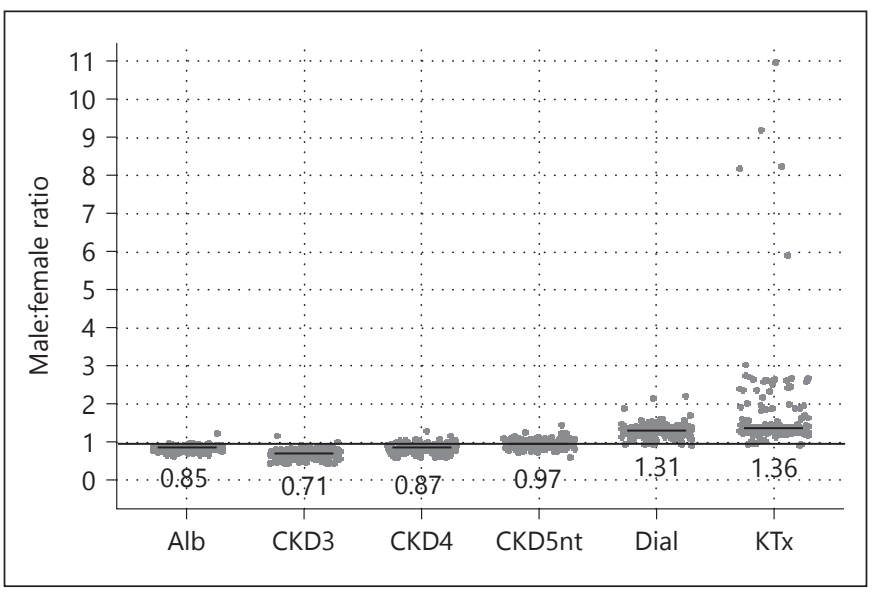

Fig. 2. Male:female ratio for each IKF condition. Each dot in the figure represents a country. For each IKF condition the median is indicated by line and numeric value. Alb, albuminuria; CKD, chronic kidney disease; Dial, dialysis; KTx, kidney transplant; IKF, impaired kidney function.

be present in 3.2 million females and 3.2 million males ( 0.8 and $0.9 \%$ from IKF respectively). Among RRT modalities, 1.3 million females and 1.7 million males were treated with dialysis, 0.3 million females and 0.4 million males had a functioning kidney graft.

The male:female all-age prevalence rate ratio significantly differed between IKF conditions $(p<0.00005)$, with values lower than 1 for albuminuria only, GFR grade 3 , GFR grade 4, and GFR grade 5 without treatment with RRT, while for dialysis and functioning kidney graft, it was higher than 1 (Fig. 2). Notably, according to the best available knowledge, RRT was not available or was almost inaccessible in many countries. Thus, in 38 countries, the dialysis prevalence rate was less than $20 \mathrm{pmp}$ and in another 15 countries, it was between 20 and $100 \mathrm{pmp}$ (19.5 and $7.7 \%$ from all world countries respectively). In 52 countries, kidney transplant recipients were absent or the prevalence rate was less than $10 \mathrm{pmp}$, and in another 26 countries, the prevalence rate was between 10 and $50 \mathrm{pmp}$ (26.7 and $13.3 \%$ from all world countries respectively).

\section{Regional and Country-Level Estimates}

A very heterogeneous picture emerged during the geospatial analysis (online suppl. Figs. S1-S5; for all online suppl. material, see www.karger.com/ doi/10.1159/000489897). In general, countries in North America and Eastern Europe tended to have a male:female ratio less than 1, while in Southern Asia and in the Middle East, this ratio was higher than 1 . But even neighbouring countries had highly contrasting male:female ratio values, while it was very similar in other, geographically distant territories. However, there were some prominent examples of extremely high male:female ratios in patients with kidney transplants in Pakistan, India and Nepal (Fig. 3), although for them GBD estimates were produced based on the limited data in the absence of national RRT registries.

\section{Correlation between CKD Prevalence and GDP}

The median male:female ratio varies substantially according to GDP per capita (online suppl. Figs. S6-S16). However, there was no uniform correlation between GDP and this ratio in all IKF conditions, and a similar male:female ratio could be observed for any IFK condition, including dialysis or kidney transplantation, in 195 countries within all ranges of GDP. The male:female ratio for either dialysis or kidney transplantation had no correlation with the GDP (online suppl. Figs. S10, S11). However, for both sexes, we found a significant and uniform correlation between GDP and ratio calculated by dividing prevalent counts of dialysis patients with a kidney transplant patients (Fig. 4). This correlation underlines the importance of economic development on the availability of kidney transplantation.

\section{Discussion}

GBD results demonstrate the substantial gender disparities in CKD prevalence, which globally was higher in women. Notably, the higher morbidity burden among females was found not only for CKD stages with decreased GFR but also for albuminuria with normal GFR. Thus, the detected higher CKD prevalence in women is a true phenomenon and cannot be attributed to the possible discrepancy between measured and eGFR due to the limitations of any eGFR equations [10]. The present findings are in line with the previous estimates of global CKD prevalence as reported in a meta-analysis with 44 studies of CKD 1-5 [11], which also showed higher CKD prevalence in women than in men. However, the magnitude of this difference is higher in our analysis, and this could be attributed to the inclusion of larger number of data sources in the GBD, eventually leading to more refined estimates. It could also be explained by the modelling process in the GBD, which assumed possible differences in the age structure of CKD population and general population of the countries. It is noteworthy that the higher prevalence of CKD in women may transfer to the next generation. Thus, $\mathrm{CKD}$ in pregnant women substantially in- 


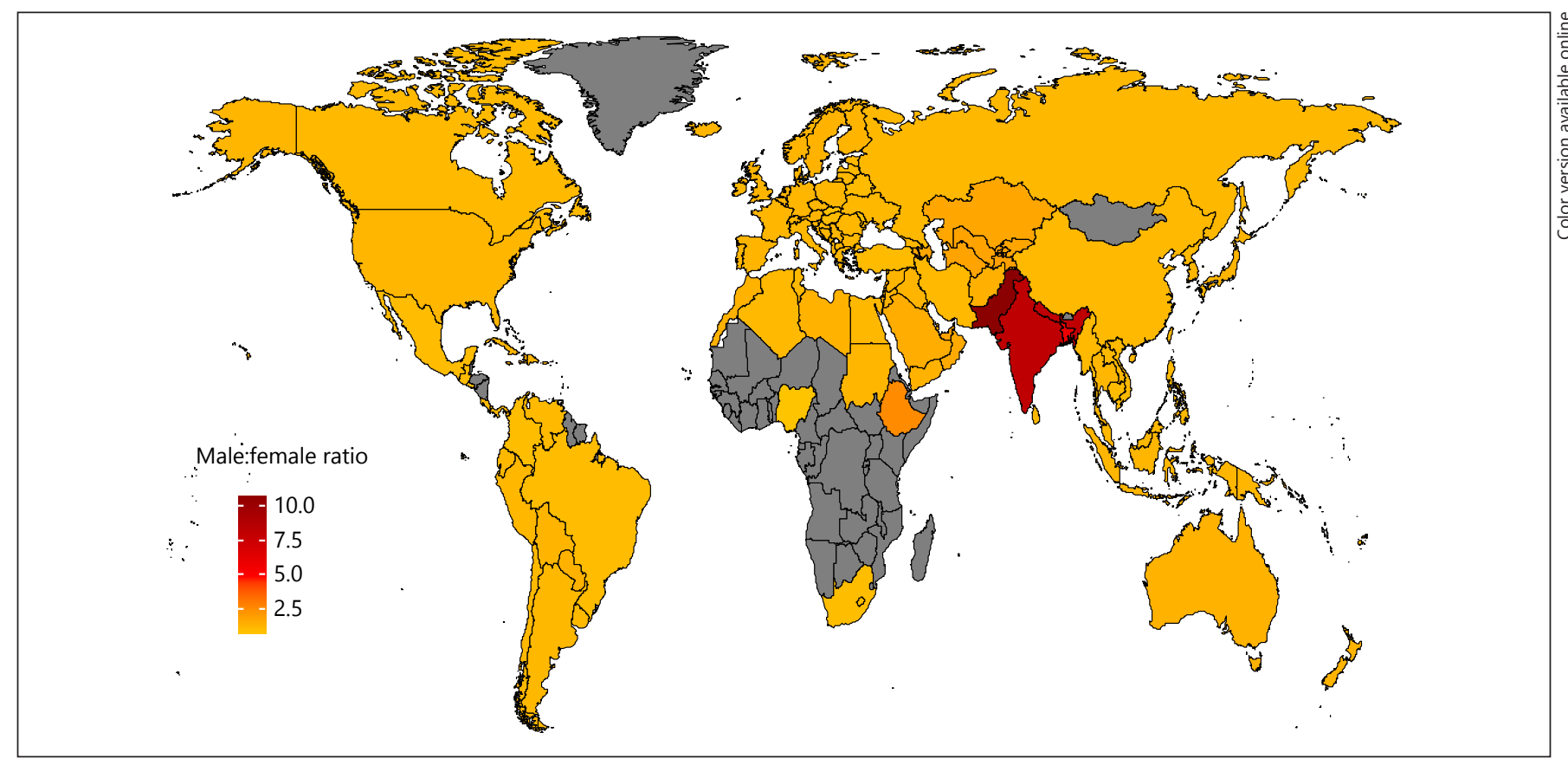

Fig. 3. Male:female ratio for the kidney transplantation prevalence rate in 120 countries with more than 50 individuals with functioning kidney graft in 2016. Grey filling marks countries with less than 50 individuals with functioning kidney graft for which the ratio is not estimated.

Fig. 4. Correlation between gross domestic product per capita and ratio of dialysis to kidney transplant prevalence count. Only countries with more than 50 patients on renal replacement therapy were included in the analysis. Each dot in the figure represents a country. GDP PPP, gross domestic product based on purchasing power parity.

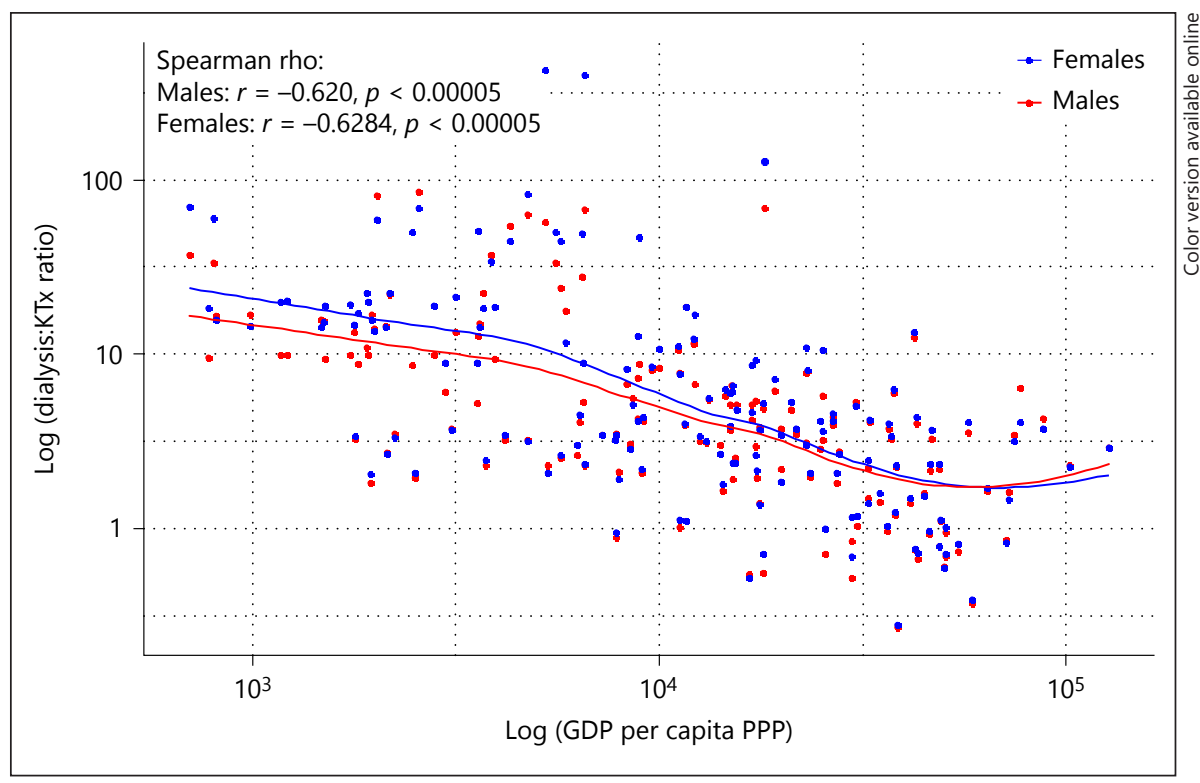

creases the odds of pre-eclampsia, premature delivery, neonates small for gestational age and low birth weight [4], which are associated with low nephron number at birth [6] - conditions that in themselves put a subject at higher risk of developing hypertension, obesity, diabetes and CKD in later life $[5,6]$. This relationship forms a desperate vicious circle that will increase the global burden of NCD in the future if no public health intervention occurs, and if current and future generations in many countries do not have access to better prevention programmes 
and universal health care. Unfortunately, recent global humanitarian crises forced by wars steal any hope there might be of improving the management of NCD, including $\mathrm{CKD}$, in vast geographical areas in almost all continents $[12,13]$. Due to this, the already high CKD prevalence in women only amplifies the morbidity burden during the collapse of social order in conflict zones.

The estimated male:female ratio for CKD that we considered reflects complex interactions between many factors, including gender differences in the prevalence of risk factors for CKD development and progression, attention to personal health care, access to medical care, availability of medications and access to RRT $[14,15]$. The latter represents one of the most sensitive indicators related to the human right for life. Unfortunately, on the global scale, the projected need for RRT remains two- to fourfold higher than its provision [16]. It is noteworthy that in many countries, a lack of universal health coverage and high out-of-pocket expenses substantially limit women's access to medical care, due to lower income, thereby resulting in lower solvency [17]. As a consequence, the treatment rate among women is lower compared to men, even in subjects with known CKD $(39.6 \%$ in women versus $45.5 \%$ in men), as was shown in a crosssectional study performed in 12 low- and middle-income countries [18]. The data regarding the CKD treatment rate in the general population of high-income countries is not available, but awareness of CKD [19] and a formal diagnosis with referrals to a nephrologist [20] was lower in women, a finding that could indicate similar general social mechanisms to the approach to gender roles across low-, middle-, and high-income countries. A constellation of extremely challenging economic conditions, as well as women's social roles as nonwage earners in some patriarchal societies may be what leads to the extremely high male:female ratio observed in our analysis in the context of dialysis and kidney transplant prevalence rates. However, this cannot be the only explanation for male predominance on RRT, since it occurs all over the world and in all GDP per capita ranges. Biological factors responsible for higher male prevalence rates on dialysis include the protective effects of estrogen in women and damaging effects of testosterone in men [15], as well as the higher prevalence of NCD risk factors in the general male population $[21,22]$ and, related to this, faster $\mathrm{CKD}$ progression rates in males [23]. The interplay between all factors may be very complex and poorly explained. As a particular example of this complexity, in the United States, the prevalence of impaired blood glucose in the general population was higher in men [21], while among haemodialysis patients, diabetes was more prevalent in women [24]. However, in Russia, Japan and countries in Western Europe, gender differences in the prevalence of NCD risk factors in the general population were directly translated into the male:female ratio in the dialysis population [22]. In cases of functioning kidney grafts, the extremely high male:female ratio in prevalent patients could be attributed to gender inequalities in the country's society with regard to accessing transplantation. However, the almost ubiquitous milder male predominance among kidney transplant patients could be explained by the fact that calculations of kidney transplant rates are performed on the whole population, without taking into account a gender-specific cohort of the population with contraindications to transplantation. Thus, women have a higher prevalence of existing panel-reactive antibodies, and adjustment to this factor could substantially level differences in sex disparities for kidney transplant rates, as shown in the United States [25].

Our analysis has limitations, with the main shortcoming related to the absence or low quality of data for many of the countries used to estimate CKD prevalence. This limitation was to some degree balanced by the GBD's advanced methods of statistical modelling, assuming the country-level covariates that facilitated the production of estimates even for countries with no primary epidemiologic data [1-3]. Moreover, since the male:female ratio is sensitive to extreme values in case of low patient numbers, and in order to avoid this, we calculated for RRT modalities the ratio both with all country data and separately with countries with substantial numbers of RRT patients, with both analyses showing similar results.

In conclusion, we demonstrated the existence of prominent gender disparities in CKD prevalence in 195 countries in the GBD Study. However, the nature of these disparities is complex and in some cases hardly explicable. Kidney transplantation could be considered a particularly prominent example of lower female access to renal health care. There is a need for substantial further work to clarify in depth the local causes of the overall gender disparities in CKD prevalence and improve the provision of universal and equal access to renal care. This would require joint efforts between the scientific community and national health authorities. What we can do as scientists is provide robust data for politicians and national health authorities to act upon, giving them evidence that their final decisions have an impact on people's health, including that of women, who are more vulnerable due to the unequal access to health care. Moreover, 
to monitor progress towards equal access in CKD prevention, diagnosis and treatment, health care authorities need clearly defined indicators that could be developed based on wide agreement in the kidney community. Current analysis provides an impact on the construction of the general framework to define such indicators and to reveal the relationships between different factors responsible for the predominance of CKD stages in different populations.

\section{Acknowledgements}

B.B. has received funding from the European Union's Horizon 2020 research and innovation programme under Marie Sklodowska-Curie grant agreement No. 703226.
The authors gratefully acknowledge all core and corresponding members of the GBD Study 2016 who assisted in estimating the burden of CKD, with special thanks to Prof. Theo Vos, Carrie Purcell and Mari Smith.

Authors thank Kerstin Mierke for her editorial assistance during preparation of the manuscript.

\section{Ethics Statement}

This study did not require informed consent nor review/approval by the appropriate ethics committee.

\section{Disclosure Statement}

The authors have no conflicts of interest to declare.

\section{References}

1 GBD 2016 Disease and Injury Incidence and Prevalence Collaborators: Global, regional, and national incidence, prevalence, and years lived with disability for 328 diseases and injuries for 195 countries, 1990-2016: a systematic analysis for the global burden of disease study 2016. Lancet 2017;390:1211-1259.

-2 GBD 2016 Causes of Death Collaborators: Global, regional, and national age-sex specific mortality for 264 causes of death, 1980-2016: a systematic analysis for the global burden of disease study 2016. Lancet 2017;390:11511210.

- 3 GBD 2016 Risk Factors Collaborators: Global, regional, and national comparative risk assessment of 84 behavioural, environmental and occupational, and metabolic risks or clusters of risks, 1990-2016: a systematic analysis for the global burden of disease study 2016. Lancet 2017;390:1345-1422.

-4 Piccoli GB, Cabiddu G, Attini R, Vigotti FN, Maxia S, Lepori N, et al: Risk of adverse pregnancy outcomes in women with CKD. J Am Soc Nephrol 2015;26:2011-2022.

5 Luyckx VA, Perico N, Somaschini M, Manfellotto D, Valensise H, Cetin I, et al: A developmental approach to the prevention of hypertension and kidney disease: a report from the low birth weight and nephron number working group. Lancet 2017;6736:1-5.

6 6 Low Birth Weight and Nephron Number Working Group: The impact of kidney development on the life course: a consensus document for action. Nephron 2017;136:3-49.

7 Piccoli GB, Alrukhaimi M, Liu ZH, Zakharova E, Levin A; World Kidney Day Steering Committee: What we do and do not know about women and kidney diseases; questions unanswered and answers unquestioned: reflection on world kidney day and international women's day. Nephron 2018; 138:249260.
8 GDP per capita, PPP (current international \$). World Bank, International Comparison Program database. https://data.worldbank. org/indicator/NY.GDP.PCAP.PP. CD?view=chart (accessed February 27, 2018).

9 KDIGO 2012 clinical practice guideline for the evaluation and management of chronic kidney disease. Kidney Int Suppl 2013;3:1150.

10 Delanaye P, Ebert N, Melsom T, Gaspari F, Mariat C, Cavalier E, et al: Iohexol plasma clearance for measuring glomerular filtration rate in clinical practice and research: a review. Part 1: how to measure glomerular filtration rate with iohexol? Clini Kidney J 2016;9:682699.

11 Hill NR, Fatoba ST, Oke JL, Hirst JA, O'Callaghan CA, Lasserson DS, et al: Global prevalence of chronic kidney disease - a systematic review and meta-analysis. PLoS One 2016;11:e0158765.

12 Perone SA, Martinez E, Mortier S, Rossi R, Pahud M, Urbaniak V, et al: Non-communicable diseases in humanitarian settings: ten essential questions. Confl Health 2017;11:17.

13 United Nations Women. Facts and Figures: Humanitarian Action. http://www.unwomen.org/en/what-we-do/humanitarian-action/ facts-and-figures (Accessed February 27, 2018).

14 Nicholas SB, Kalantar-zadeh K, Norris KC: Socioeconomic disparities in chronic kidney disease. Adv Chronic Kidney Dis 2018;22:6-15.

15 Carrero JJ, Hecking M, Chesnaye NC, Jager $\mathrm{KJ}$ : Sex and gender disparities in the epidemiology and outcomes of chronic kidney disease. Nat Rev Nephrol 2018;14:151-164. 16 Liyanage T, Ninomiya T, Jha V, Neal B, Pa-
trice HM, Okpechi I, et al: Worldwide access to treatment for end-stage kidney disease: a systematic review. Lancet 2015 Mar 11;385: 1975-1982.
17 Carrero J, Hecking M, Ulasi I: Chronic kidney disease, gender, and access to care: a global perspective. Semin Nephrol 2017;37:296-308.

18 Ene-Iordache B, Perico N, Bikbov B, Carminati S, Remuzzi A, Perna A, et al: Chronic kidney disease and cardiovascular risk in six regions of the world (ISN-KDDC): a cross-sectional study. Lancet Glob Heal 2016; 4:e307-e319.

19 Coresh J, Byrd-Holt D, Astor BC, Briggs JP, Eggers PW, Lacher DA, et al: Chronic kidney disease awareness, prevalence, and trends among U.S. adults, 1999 to 2000. J Am Soc Nephrol 2005;16:180-188.

20 Gasparini A, Evans M, Coresh J, Grams ME, Norin O, Qureshi AR, et al: Prevalence and recognition of chronic kidney disease in Stockholm healthcare. Nephrol Dial Transpl 2016;31:2086-2094.

21 World Health Statistics. Geneva, WHO, 2014.

22 Bikbov B, Bieber B, Andrusev A, Tomilina N, Zemchenkov A, Zhao J, et al.: Hemodialysis practice patterns in the Russia dialysis outcomes and practice patterns study (DOPPS), with international comparisons. Hemodial Int 2017;21:393-408.

23 Neugarten J, Acharya A, Silbiger SR: Effect of gender on the progression of nondiabetic renal disease: a meta-analysis. J Am Soc Nephrol 2000;11:319-329.

24 Hecking M, Bieber BA, Ethier J, KautzkyWiller A, Sunder-Plassmann G, Säemann $\mathrm{MD}$, et al: Sex-specific differences in hemodialysis prevalence and practices and the maleto-female mortality rate: the dialysis outcomes and practice patterns study (DOPPS). PLoS Med 2014;11:e1001750.

25 Wolfe RA, Ashby VB, Milford EL, Bloembergen WE, Agodoa LY, Held PJ, et al: Differences in access to cadaveric renal transplantation in the United States. Am J Kidney Dis 2000;36:1025-1033. 